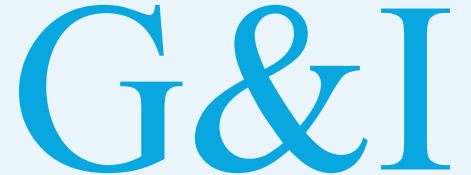

Genomics \& Informatics

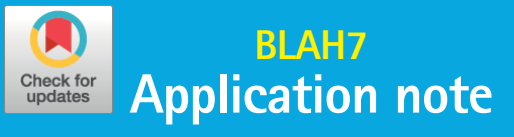

elSSN 2234-0742

Genomics Inform 2021;19(3):e21

https://doi.org/10.5808/gi.21011

Received: March 12, 2021

Accepted: July 26, 2021

${ }^{*}$ Corresponding author:

E-mail: jbanda@gsu.edu

\section{A biomedically oriented automatically annotated Twitter COVID-19 dataset}

\author{
Luis Alberto Robles Hernandez', Tiffany J. Callahan ${ }^{2}$, Juan M. Banda ${ }^{1 *}$ \\ 1 Department of Computer Science, Georgia State University, Atlanta, GA 30303, USA \\ ${ }^{2}$ Computational Bioscience Program, University of Colorado Anschutz Medical Campus, \\ Aurora, C0 80045, USA
}

The use of social media data, like Twitter, for biomedical research has been gradually increasing over the years. With the coronavirus disease 2019 (COVID-19) pandemic, researchers have turned to more non-traditional sources of clinical data to characterize the disease in near-real time, study the societal implications of interventions, as well as the sequelae that recovered COVID-19 cases present. However, manually curated social media datasets are difficult to come by due to the expensive costs of manual annotation and the efforts needed to identify the correct texts. When datasets are available, they are usually very small and their annotations don't generalize well over time or to larger sets of documents. As part of the 2021 Biomedical Linked Annotation Hackathon, we release our dataset of over 120 million automatically annotated tweets for biomedical research purposes. Incorporating best-practices, we identify tweets with potentially high clinical relevance. We evaluated our work by comparing several SpaCy-based annotation frameworks against a manually annotated gold-standard dataset. Selecting the best method to use for automatic annotation, we then annotated 120 million tweets and released them publicly for future downstream usage within the biomedical domain.

Keywords: biomedical annotations, COVID-19, datasets, social media data

Availability: All code and documentation related to this project are publicly available on GitHub (https://github.com/thepanacealab/ annotated_twitter_covid19_dataset).

\section{Introduction}

Social media platforms like Twitter, Instagram, and Facebook provide researchers with unprecedented insight into personal behavior on a global scale. Twitter is currently one of the leading social networking services with over 353 million users and reaching $\sim 6 \%$ of the world's population over the age of 13 [1]. It is also quickly becoming one of the most popular platforms for conducting health-related research because of its use for public health surveillance, pharmacovigilance, event detection/forecasting, and disease tracking [2,3]. During the last decade, Twitter has provided substantial aid in the surveillance of pandemics, including the Zika virus [4], H1N1 (or Swine Flu) [5], H7N9 (or avian/bird flu) [6], and Ebola [7]. Twitter has been used extensively during the 2020 coronavirus disease 2019 (COVID-19) outbreak [8], providing insight into everything from monitoring communication between public health officials and world leaders [9], tracking emerging symptoms $[10]$ and access to testing facilities [11], to understanding the public's top fears and concerns about infection rates and vaccination [12]. While it is clear that Twitter contains invaluable content that can be used for a myriad of benevolent endeavors, there are many challenges to accessing and leveraging these data for clinical research and/or applications.

Researchers face a myriad of challenges when trying to utilize Twitter data. Aside from
2021 Korea Genome Organization

(c) This is an open-access article distributed under the terms of the Creative Commons Attribution license (http://creativecommons. org/licenses/by/4.0/), which permits unrestricted use, distribution, and reproduction in any medium, provided the original work is properly cited 
the potential ethical challenges, which will not be discussed in this work (see Webb et al. [13] for a review of this area), it can be difficult to obtain access to these data and hard to keep up with real-time content collection $[14,15]$. Once the data have been obtained, researchers must then perform several preprocessing steps to ensure the data are sufficient for analysis. Concerning COVID-19, there are several existing social media repositories [16-20]. Unfortunately, most of these repositories are infrequently updated, do not provide any preprocessing or data cleaning, and either do not provide the raw data or lack appropriate metadata or provenance. The COVID-19 Twitter Chatter dataset [20] is a robust large-scale repository of tweets that is well-maintained and frequently updated (over 50 versions released at the time of publication). Recent work utilizing this resource has shown great promise for tracking longterm patient-reported symptoms [21] as well as highlighted mentions of drugs relevant to the treatment of COVID-19 [22]. While these are compelling clinical use cases, additional work is needed to fully understand what additional biomedical and clinical utility can be obtained from these data.

This paper presents preliminary work achieved during the 2021 Biomedical Linked Annotation Hackathon (BLAH 7) [23], which aimed to enhance and extend the COVID-19 Twitter Chatter dataset $[20]$ to include biomedical entities. By annotating symptoms and other relevant biomedical entities from COVID-19 tweets, we hope to improve the downstream clinical utility of these data and provide researchers with a means to clinically characterize personally-reported COVID-19 phenomena. We envision this work as the first step towards our larger goal of deriving mechanistic insights from specific types of entities within COVID-19 tweets by integrating these data with larger and more complex sources of biomedical knowledge, like PheKnowLator [24] and the KG-COVID-19 [25] knowledge graphs. The remainder of this paper is organized as follows: an overview of the methods and technologies utilized in this work, an overview of our findings, and a brief discussion of conclusions and future work.

\section{Methods}

To prepare the dataset released in this work, we looked for named entity recognition (NER) pipelines to identify biomedical entities in text. We opted to evaluate: MedSpaCy [26], MedaCy [27], and ScispaCy [28], alongside a traditional text annotation pipeline from Social Media Mining Toolkit (SMMT), a product of a BLAH 6 hackathon [29]. The main reason for selecting these text processing pipelines is the fact that they are all based on $\mathrm{SpaCy}$ [30], a widely adopted open-source library for Natural Language Processing (NLP) in Python, allowing our codebases to be streamlined, and the annotation output to be easily compared in our evaluation as well as ingested by other work utilizing similar pipelines. Several preprocessing steps like URL and emoji removal were performed on all tweets.

Please note that the selected NER pipelines are usually tuned and developed to annotate specific types of clinical/scientific text, from either electronic health records, clinical notes, or scientific literature. The only general-purpose tagger is the SMMT, which does not perform any specialized tasks other than tagging or annotating text. This fact impacted their performance in Twitter social media data, and the following comparison should not be used to evaluate the systems' performance on clinical data/scientific literature, but rather the need for appropriately tuned systems for social media data.

\section{Datasets}

As the source for this work, we used one of the largest COVID-19 Twitter Chatter datasets available [20]. We used version 44 of the dataset [20], which contains $903,223,501$ unique tweets. To improve the quality and relevance of the annotations, we used the clean version of this dataset, which has all retweets removed. Leaving us with a total of 226,582,903 unique tweets to annotate. From this subset, we selected only English tweets, as all the systems evaluated were created to extract/annotate biomedical concepts in this language.

For the evaluation of the annotations from each NER system and the SMMT tagger, we will use as a gold standard, a manually annotated dataset created for symptoms, conditions, prescriptions, and measurement procedures identification in patients with long Covid phenotypes [21]. This dataset consists of 10,315 manually annotated tweets, by multiple clinicians. Currently, the dataset is not publicly available but will be released at a later date.

\section{ScispaCy}

Developed by the Allen AI institute, the pipelines and models in this package have been tuned for use on scientific documents [28]. In our evaluation, we used the following model: en_core_sci_lg, which consists of $\sim 785 \mathrm{k}$ vocabulary and $600 \mathrm{k}$ word vectors. Additionally, we used the EntityLinker component to annotate the Unified Medical Language System (UMLS) concepts. Since this pipeline provides more than one match per annotation, we only selected the first match to avoid duplicates. The code used can be found in [31].

\section{MedaCy}

Developed by researchers at Virginia Commonwealth University, $\mathrm{MedaCy}$ is a text processing framework wrapper for spaCy. It supports extremely fast prototyping of highly predictive medical NLP models. For our evaluation, we used their provided medacy_model_ 
clinical_notes model, with all other default settings. The code used can be found [31].

\section{MedSpaCy}

Currently, in beta release, MedSpaCy was created as a toolkit to enable user-specific clinical NLP pipelines. In our evaluation, we wanted to use some of the out-of-the-box components instead of fine-tuning them for our Twitter annotation task. We used the en info_3700_2b2_2012 model - trained on i2b2 data, and the Sectionizer [32]. We initially tried to use the demo QuickUMLS entity linker, but ultimately opted not to do this as their demo only includes 100 concepts, and building it from scratch was outside of the scope of our task. The code used can be found in [31].

\section{SMMT tagger}

As part of SMMT, the SpaCy-based tagger relies on a user-specified dictionary to annotate concepts on the provided text. This tagger does not perform any NER or section detection, but only simple string matching. Designed with simplicity and flexibility in mind, when using social media data, it is preferred to provide a concise dictionary with the desired terms for annotation, rather than using pre-trained models that may not generalize well to domain-specific tasks, or are computationally expensive. The dictionary used in this evaluation consists of a mix of SNOMED-CT [33], ICD 9/10 [34], MeSH [35], and RxNorm [36] extracted from the Observational Health Data Sciences and Informatics (OHDSI) vocabulary. This dictionary is available as part of the paper's code repository.

\section{Results}

\section{Extraction performance}

In Table 1 we show the processing time and count of annotations produced by the evaluated systems on the gold standard dataset. Note that as expected, simple text annotation from the SMMT tagger is the fastest, with MedaCy coming in second as its annotation model is small. The SMMT tagger dictionary produces plenty of annotations as it considers some of the common misspellings for COVID-19 (e.g., "fatigue" vs "fatige") as well as related symptoms and drugs that have been curated in our previous work when ex-

Table 1. Extraction evaluation of proposed systems

\begin{tabular}{lccc}
\hline & Tweets & Annotations produced & Processing time (s) \\
\hline SMMT Tagger & 10,315 & 92,835 & $10,815.24$ \\
MedSpaCy & 10,315 & 51,575 & $33,746.40$ \\
MedaCy & 10,315 & 61,890 & $21,896.63$ \\
ScispaCy & 10,315 & 72,205 & $49,168.85$ \\
\hline
\end{tabular}

SMMT, Social Media Mining Toolkit. tracting drug mentions in Twitter data [22].

Due to the larger model utilized by ScispaCy, the processing time is nearly five-fold that of simple text annotation. However, this comes with the added benefit that abbreviations are nicely normalized to UMLS concepts, hence creating some annotations that any of the other systems will be unable to find.

\section{Overlap between systems on gold standard dataset}

To determine which system to use for the large-scale annotation of the Twitter COVID-19 chatter dataset, we evaluated all systems against the manually annotated gold-standard. Here, while we grouped the annotations into three categories: drugs, conditions/ symptoms, and measurements. We did not use the systems' annotation categories, but rather their annotated terms and spans. This was done to accommodate the custom entity categories that systems like MedSpaCy and MedaCy have in their default settings and the fact that we are using only the first UMLS concepts identified by ScispaCy. Table 2 shows the annotation overlap analysis.

We would like to stress again that MedSpaCy and MedaCy are at a disadvantage as their models are trained on considerably different data that does not work well with Twitter data. ScispaCy, however, performs fairly decently (in comparison) as the larger models provide capture relevant annotations when the tweet's text is clean and well-formed. It is out of the scope of this paper to properly tune these systems to ensure that they perform well with Twitter data, but it is certainly an interesting avenue for future research.

\section{Extraction evaluation on a limited set}

While it is clear that regular text annotation performed the best in replicating the annotations that our clinicians made, we still annotated all 226,582,903 dataset tweets and evaluated the overlap of annotations made by the different systems. Table 3 shows the comparison between counts of produced annotations, processing time, and overlaps in annotations between the systems.

\section{Conclusion}

In this work we release a biomedically oriented automatically anno-

Table 2. Annotation overlap analysis between gold standard dataset and evaluated systems

\begin{tabular}{lcccc}
\hline & Drugs (\%) & $\begin{array}{c}\text { Conditions/ } \\
\text { Symptoms (\%) }\end{array}$ & Measurements (\%) & Average (\%) \\
\hline SMMT Tagger & 69.31 & 71.91 & 39.83 & 60.35 \\
MedSpaCy & 19.98 & 13.49 & 7.45 & 13.64 \\
MedaCy & 47.04 & 27.14 & 12.56 & 28.91 \\
ScispaCy & 59.71 & 44.65 & 26.98 & 43.78 \\
\hline
\end{tabular}

SMMT, Social Media Mining Toolkit. 
Table 3. Annotation overlap evaluation for complete dataset

\begin{tabular}{lcccccc}
\hline & $\begin{array}{c}\text { Annotations } \\
\text { produced }\end{array}$ & Processing time $(\mathrm{min})$ & $\begin{array}{c}\text { Overlaps with SMMT } \\
(\%)\end{array}$ & $\begin{array}{c}\text { Overlap with } \\
\text { MedSpaCy (\%) }\end{array}$ & $\begin{array}{c}\text { Overlap with } \\
\text { MedaCy (\%) }\end{array}$ & $\begin{array}{c}\text { Overlap with } \\
\text { ScispaCy (\%) }\end{array}$ \\
\hline SMMT Tagger & $751,245,366$ & 24,120 & 100 & 20.12 & 33.91 & 72.28 \\
MedSpaCy & $582,768,145$ & 159,267 & 53.48 & 100 & 42.23 & 55.39 \\
MedaCy & $656,311,799$ & 26,147 & 51.14 & 44.92 & 100 & 49.73 \\
ScispaCy & $775,615,621$ & 325,620 & 89.17 & 34.77 & 44.17 \\
\hline
\end{tabular}

SMMT, Social Media Mining Toolkit.

tated dataset of COVID-19 chatter tweets. We demonstrate that while there are existing SpaCy-based systems for NER on clinical and scientific documents, they do not generalize well when used on non-clinical sources of data like tweets. However, we use this evaluation to justify the usage of a simple text tagger (SMMT) to produce annotations on a large set of tweets, based on its robustness when evaluated on a gold-standard manually curated dataset. The resulting dataset and biomedical annotations is the first and largest of its kind making it a substantial contribution with respect to using large-scale Twitter data for biomedical research. We have also added components for these types of tasks to SMMT, improving the usability of the resource.

As for future work, the release of this dataset will facilitate continued development of fine-tuned resources for mining social media data for biomedical and clinical applications. Recent research has shown social media data to be a valuable source of patient-reported information that is not available in similar granularity in other more traditional data sources.

\section{ORCID}

Luis Alberto Robles Hernandez:

https://orcid.org/0000-0002-5396-7800

Tiffany J. Callahan: https://orcid.org/0000-0002-8169-9049

Juan M. Banda: https://orcid.org/0000-0001-8499-824X

\section{Authors' Contribution}

Conceptualization: JMB, TJC. Data curation: JMB, LARH. Formal analysis: JMB, TJC. Methodology: JMB, LARH, TJC. Writing original draft: JMB, TJC, LARH. Writing - review \& editing: JMB, TJC, LARH.

\section{Conflicts of Interest}

No potential conflict of interest relevant to this article was reported.

\section{Acknowledgments}

We would like to thank Jin-Dong Kim and the organizers of the virtual Biomedical Linked Annotation Hackathon 7 for providing us a space to work on this project and their valuable feedback during the online sessions.

\section{References}

1. Newberry C. 36 Twitter statistics all marketers should know in 2021. Vancouver: Hootsuite Inc., 2021. Accessed 2021 Mar 9. Available from: https://blog.hootsuite.com/twitter-statistics/.

2. Sinnenberg L, Buttenheim AM, Padrez K, Mancheno C, Ungar L, Merchant RM. Twitter as a tool for health research: a systematic review. Am J Public Health 2017;107:e1-e8.

3. Edo-Osagie O, De La Iglesia B, Lake I, Edeghere O. A scoping review of the use of Twitter for public health research. Comput Biol Med 2020;122:103770.

4. Masri S, Jia J, Li C, Zhou G, Lee MC, Yan G, et al. Use of Twitter data to improve Zika virus surveillance in the United States during the 2016 epidemic. BMC Public Health 2019;19:761.

5. Chew C, Eysenbach G. Pandemics in the age of Twitter: content analysis of Tweets during the 2009 H1N1 outbreak. PLoS One 2010;5:e14118.

6. Vos SC, Buckner MM. Social media messages in an emerging health crisis: Tweeting bird flu. J Health Commun 2016;21:301308.

7. Tang L, Bie B, Park SE, Zhi D. Social media and outbreaks of emerging infectious diseases: a systematic review of literature. Am J Infect Control 2018;46:962-972.

8. Coronavirus: staying safe and informed on Twitter. San Francisco: Twitter Inc., 2021. Accessed 2021 Mar 9. Available from: https:// blog.twitter.com/en_us/topics/company/2020/covid-19.html.

9. Rufai SR, Bunce C. World leaders' usage of Twitter in response to the COVID-19 pandemic: a content analysis. J Public Health (Oxf) 2020;42:510-516.

10. Guo JW, Radloff CL, Wawrzynski SE, Cloyes KG. Mining twitter to explore the emergence of COVID-19 symptoms. Public 
Health Nurs 2020;37:934-940.

11. Mackey T, Purushothaman V, Li J, Shah N, Nali M, Bardier C, et al. Machine learning to detect self-reporting of symptoms, testing access, and recovery associated with COVID-19 on Twitter: retrospective big data infoveillance study. JMIR Public Health Surveill 2020;6:e19509.

12. Abd-Alrazaq A, Alhuwail D, Househ M, Hamdi M, Shah Z. Top concerns of Tweeters during the COVID-19 pandemic: infoveillance study. J Med Internet Res 2020;22:e19016.

13. Webb H, Jirotka M, Stahl BC, Housley W, Edwards A, Williams $\mathrm{M}$, et al. The ethical challenges of publishing Twitter data for research dissemination. In: Proceedings of the 2017 ACM on Web Science Conference, 2017 Jun 25-28, Troy, NY, USA. New York: Association for Computing Machinery, 2017.pp. 339-348.

14. Hino A, Fahey RA. Representing the Twittersphere: archiving a representative sample of Twitter data under resource constraints. Int J InfManage 2019;48:175-184.

15. Kim Y, Nordgren R, Emery S. The story of goldilocks and three Twitter's APIs: a pilot study on Twitter data sources and disclosure. Int J Environ Res Public Health 2020;17:864.

16. Kabir MY, Madria S. CoronaVis: a real-time COVID-19 Tweets data analyzer and data repository. Preprint at: https://arxiv.org/ abs/2004.13932 (2020).

17. Chen E, Lerman K, Ferrara E. Tracking social media discourse about the COVID-19 pandemic: development of a public coronavirus Twitter data set. JMIR Public Health Surveill 2020;6: e19273.

18. Gupta RK, Vishwanath A, Yang Y. Global reactions to COVID-19 on Twitter: a labelled dataset with latent topic, sentiment and emotion attributes. Preprint at: http://arxiv.org/abs/2007.06954 (2021).

19. Alqurashi S, Alhindi A, Alanazi E. Large arabic Twiter dataset on COVID-19. Preprint at: https://arxiv.org/abs/2004.04315 (2020).

20. Banda JM, Tekumalla R, Wang G, Yu J, Liu T, Ding Y, et al. A large-scale COVID-19 Twitter chatter dataset for open scientific research: an international collaboration. Epidemiologia 2021;2: 315-324.

21. Banda JM, Singh SR, Alser OH, Prieto-Alhambra D. Long-term patient-reported symptoms of COVID-19: an analysis of social media data. Preprint at: https://doi.org/10.1101/2020.07. 29.20164418 (2020).

22. Tekumalla R, Banda JM. Characterizing drug mentions in COVID-19 Twitter Chatter. New York: Association for Computational Linguistics, 2020. Accessed 2021 Mar 9. Available from: https://www.aclweb.org/anthology/2020.nlpcovid19-2.25/.

23. Biomedical Linked Annotation Hackathon 7. Kashiwa: Database Center for Life Science, 2021. Accessed 2021 Mar 9. Available from: https://blah7.linkedannotation.org/.

24. Callahan TJ, Tripodi IJ, Hunter LE, Baumgartner WA Jr. KGCOVID-19: a framework to produce customized knowledge graphs for COVID-19 response. Preprint at: https://doi.org/10. 1101/2020.04.30.071407 (2020).

25. Reese JT, Unni D, Callahan TJ, Cappelletti L, Ravanmehr V, Carbon S, et al. KG-COVID-19: a framework to produce customized knowledge graphs for COVID-19 response. Patterns (N Y) 2021;2:100155.

26. medspacy. San Francisco: GitHub, 2021. Accessed 2021 Mar 9. Available from: https://github.com/medspacy/medspacy.

27. Mulyar A, Mahendran D, Maffey L, Olex A, Matteo G, Dill N, et al. TAC SRIE 2018: extracting systematic review information with MedaCy. Gaithersburg: National Institute of Standards and Technology, 2018. Accessed 2021 Mar 9. Available: https://www. researchgate.net/profile/Darshini_Mahendran/publication/ 340870892_TAC_SRIE_2018_Extracting_Systematic_Review_Information_with_MedaCy/links/5ealadd5a6fdcc88fc381e4c/TAC-SRIE-2018-Extracting-Systematic-Review-Information-with-MedaCy.pdf.

28. Neumann M, King D, Beltagy I, Ammar W. ScispaCy: fast and robust models for biomedical natural language processing. New York: Association for Computational Linguistics, 2019. Accessed 2021 Mar 9. https://doi.org/10.18653/v1/W19-5034.

29. Tekumalla R, Banda JM. Social Media Mining Toolkit (SMMT). Genomics Inform 2020;18:e16.

30. Explosion AI. spaCy-Industrial-strength Natural Language Processing in Python. Explosion AI, 2017. Accessed 2021 Mar 9. Available from: https://spacy.io/.

31. Annotated_twitter_covid19_dataset. San Francisco: Github, 2021. Accessed 2021 Mar 9. Available from: https:// github.com/ thepanacealab/annotated_twitter_covid19_dataset.

32. medspacy. San Francisco: Github, 2021. Accessed 2021 Mar 9. Available from: https://github.com/medspacy/medspacy.

33. Donnelly K. SNOMED-CT: the advanced terminology and coding system for eHealth. Stud Health Technol Inform 2006;121: 279-290.

34. International Statistical Classification of Diseases and Related Health Problems (ICD). Geneva: World Health Organization, 2020. Accessed 2021 Mar 10. Available from: https://www.who. int/standards/classifications/classification-of-diseases.

35. Medical subject headings. Bethesda: National Library of Medicine, 2020. Accessed 2021 Mar 10. Available from: https:// www. nlm.nih.gov/mesh/meshhome.html.

36. RxNorm. Bethesda: National Library of Medicine, 2004. Accessed 2021 Mar 10. Available from: https:/www.nlm.nih.gov/ research/umls/rxnorm/index.html. 